\title{
Incentivize Crowd Labeling under Budget Constraint
}

\author{
Qi Zhang*, Yutian Wen*, Xiaohua Tian*, Xiaoying Gan*, Xinbing Wang ${ }^{\dagger \ddagger}$ \\ *Department of Electronic Engineering, Shanghai Jiao Tong University, Shanghai, China \\ ${ }^{\dagger}$ School of Electronic, Info. \& Electrical Engineering, Shanghai Jiao Tong University, Shanghai, China

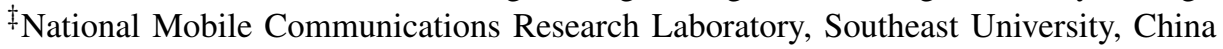 \\ Email: $\{362802781$, nogerw, xtian, ganxiaoying, xwang8\}@sjtu.edu.cn
}

\begin{abstract}
Crowdsourcing systems allocate tasks to a group of workers over the Internet, which have become an effective paradigm for human-powered problem solving such as image classification, optical character recognition and proofreading. In this paper, we focus on incentivizing crowd workers to label a set of binary tasks under strict budget constraint. We properly profile the tasks' difficulty levels and workers' quality in crowdsourcing systems, where the collected labels are aggregated with sequential Bayesian approach. To stimulate workers to undertake crowd labeling tasks, the interaction between workers and the platform is modeled as a reverse auction. We reveal that the platform utility maximization could be intractable, for which an incentive mechanism that determines the winning bid and payments with polynomial-time computation complexity is developed. Moreover, we theoretically prove that our mechanism is truthful, individually rational and budget feasible. Through extensive simulations, we demonstrate that our mechanism utilizes budget efficiently to achieve high platform utility with polynomial computation complexity.
\end{abstract}

\section{INTRODUCTION}

In recent years, online crowdsourcing systems such as Amazon Mechanical Turk (MTurk) have become flourishing for human computation. The paradigm of crowdsourcing provides access to a group of workers over the Internet who are available to solve problems such as image labeling, optical character recognition and proofreading. Projects like reCAPTCHA [1] have made it possible to harness human resources solving learning problems. MTurk establishes a market where a "crowdsourcer" can submit batches of small tasks, and any workers can pick them up in exchange for monetary rewards.

Incentivizing adequate workers to undertake labeling tasks is crucial to the system. Several platforms provide workers with non-monetary incentives like entertainment [2], [3]; however, incentivizing the crowd with monetary payments is more effective [4], [5], [6], [7], where the task allocation and pricing are vitally important. Since the crowdsourcer often faces budget constraints and workers are diverse in their skills and backgrounds, designing effective allocation and pricing schemes is non-trivial. Karger et al. allocate tasks with a random bipartite graph [8]. However, they first assume that the platform can access any worker repeatedly, which is not true in practice. Secondly, they merely price each task with equal amount of monetary rewards, which is not enough to attract diverse workers. Yang et al. propose two types of incentive mechanisms for maximizing the utility of the mobile sensing applications [9]. Incentive mechanisms for mobile crowd sensing can hardly be applied to the crowd labeling system, where it is inappropriate to evaluate the contribution of the workers by working time.

In practice, the labels provided by the crowd could be very noisy. On one hand, the crowd is anonymous and transient. They may arbitrarily submit their labeling answers oblivious to the question, therefore get rewards free of effort by providing useless labels. On the other hand, crowd workers are of different levels of expertise and tasks may vary in difficulty levels. Some workers may provide more reliable labeling answers than others. It is still an open question how to effectively aggregate collected labels. Karger et al. use a probabilistic model to analyze the crowd quality and aggregate collected labels with low-rank approximation [10]. Raykar et al. manage to improve the labeling accuracy by discriminating workers with scores [11]. These label aggregation methods, however, do not mention how the incentive mechanisms should be designed correspondingly.

In this paper, we design a truthful and budget feasible mechanism to incentivize crowd workers to undertake the binary labeling tasks, where the labeling answer is either 0 or 1 . The interaction between the platform and the workers is modeled as a reverse auction. The platform publicizes a set of labeling tasks, after which workers can submit bids for a subset of tasks according to their preference. For performing a task, each worker has a private cost and claims it publicly in the bids. The platform then determines the set of winning bids by which the labeling tasks are allocated to the worker. Each winning bid is paid with an amount of money in exchange for the labeling answer.

The mechanism mainly consists of winning bid allocation scheme and payment scheme, which together guarantee truthfulness, individual rationality, and budget feasibility. With our mechanism, each worker's optimal strategy is to disclose the true cost in his bids. Moreover, the total payment determined by the mechanism is under strict budget constraint for the sake of the crowdsourcer. The mechanism is individual rational in the sense that the payment must cover the cost. To profile the labeling difficulty, we propose to associate each task with a soft label, which is the probability that the task is labeled as 1 by a reliable worker [12]. To recognize the quality of 
crowd workers, we propose to use the one-coin model. The Bayesian sequential approach is applied to update the soft labels and quality parameters based on crowd answers, so that the platform determines the true answers of tasks by calculating the posterior with collected labels. The platform utility is defined as the difference between the prior and the posterior of all soft labels.

Adapting label aggregation into the reverse auction based mechanism is non-trivial. To this end, we overcome the following two challenges: 1 ) The platform utility maximization can be formulated into a Markov Decision Process (MDP), the optimal solution of which can be found by backward induction that is mathematically intractable. 2) The payment scheme should guarantee truthfulness and budget feasibility simultaneously. We design a winning bid allocation scheme that is computational efficient. For better representation, we first assume the workers are uniformly perfect. Then we further apply our allocation scheme to the more general case where the workers are of diverse quality. Our payment scheme is developed by adapting the budget feasible mechanism proposed by Singer [13].

We summarize our contributions as follows.

- We model the interaction between the platform and crowd workers as a reverse auction in the setting of binary labeling tasks. We introduce the soft label and the quality parameter to profile tasks and workers, respectively. The maximization of the platform utility is formulated into a Markov Decision Process whose optimal solution is computationally intractable.

- We design a winning bid allocation algorithm for uniform, and diverse workers, respectively. The algorithm runs in polynomial time to achieve computational efficiency. We then present our payment scheme to guarantee truthfulness, individual rationality, and budget feasibility.

The rest of the paper is organized as follows. Section II presents the system model, the reverse auction framework and label aggregation to formulate the mathematical problem. Then, we propose our mechanism in Section III. The theoretical analysis of the proposed mechanism is presented in Section IV. In Section V, we evaluate the performance of our mechanism. Section VI reviews the related work and Section VII concludes the paper.

\section{System Model and Problem Formulation}

\section{A. Reverse Auction Framework}

We are interested in predicting true labels of a fixed set of binary tasks, by requiring a fixed group of crowd workers. In the crowdsourcing system, the interaction between the platform and the crowd workers can be modeled as a reverse auction. Crowd workers sell their wisdom to the platform by providing their labeling answers. The auction framework is described as follows.

1) The platform publicizes a set $\mathcal{M}=\left\{t_{1}, t_{2}, \ldots, t_{M}\right\}$ of binary labeling tasks to a set of $\mathcal{N}=\{1,2, \ldots, N\}$ crowd workers.
2) Each worker $j \in \mathcal{N}$ replies with a set $\Theta_{j}$ of $k_{j}$ bids, each of which is a task-price pair $\theta_{j}^{k}=\left(t_{j}^{k}, b_{j}^{k}\right)$, where $t_{j}^{k}$ is a single task from $\mathcal{M}$, and $b_{j}^{k}$ is the charge worker $j$ claims for performing $t_{j}^{k}$.

3) The platform sequentially determines the winning bid set, $\Theta_{\mathcal{W}} \subseteq \Theta=\bigcup_{j \in N} \Theta_{j}$, from all submitted bids. It selects a bid into the current winning bid set, observe the labeling answer, and update the posterior distributions before selecting the next one.

4) Each winning bid, $\theta_{j}^{k} \in \Theta_{\mathcal{W}}$, is paid an amount of money $p\left(\theta_{j}^{k}\right)$ by the platform. The platform infers true labels according to the posterior distributions of the soft label.

Meanwhile, each bid $\theta_{j}^{k}$ has an associated cost $c\left(t_{j}^{k}\right) \in \mathbb{R}_{+}$. The cost of performing the task $t_{j}^{k}$ is private and only known to worker $j$. Each worker strategically determines $b_{j}^{k}$ to maximizing his own utility. We define the utility of a worker as follows.

Definition 1(Worker's Utility). The utility of a worker is defined as the difference between the total payment it receives and his total cost.

$$
u_{j}=\sum_{\theta_{j}^{k} \in \Theta_{\mathcal{W}}}\left(p\left(\theta_{j}^{k}\right)-c\left(t_{j}^{k}\right)\right)
$$

\section{B. Aggregating Labels from Perfect Workers}

The platform publicizes a set $\mathcal{M}=\left\{t_{1}, t_{2}, \ldots, t_{M}\right\}$ of binary labeling tasks, and employs a set $\mathcal{N}=\{1,2, \ldots, N\}$ of crowd workers to label them. Each task is associated with a true label $z_{i} \in\{0,1\}, i=1,2, \ldots, M$. We denote a labeling answer from a crowd worker $j$ to task $t_{i}$ as $y_{i j} \in\{0,1\}$. The platform view $y_{i j}$ as a random variable $Y_{i j}$ before the inquiry.

A soft label $\vartheta_{i} \in[0,1]$ is used to measure the labeling difficulty of task $t_{i}$. It is defined as the probability that the task is labeled as 1 by a perfectly reliable worker, i.e.

$$
\operatorname{Pr}\left(Y_{i j}=y \mid \vartheta_{i}\right)=\left(\vartheta_{i}\right)^{y}\left(1-\vartheta_{i}\right)^{1-y}
$$

which is Bernoulli distribution of parameter $\vartheta_{i}$. Since the soft labels are defined on the interval $[0,1]$, we set a priori distribution on $\vartheta_{i} \sim \operatorname{Beta}\left(a_{i}^{0}, b_{i}^{0}\right)$ as done in [12]. The probability density function (PDF) of the Beta distribution with parameter $(a, b)$ is $\operatorname{Beta}(x ; a, b)=\frac{1}{\mathrm{~B}(a, b)} x^{a-1}(1-x)^{b-1}, x \in[0,1]$, where $\mathrm{B}(a, b)=\frac{\Gamma(a) \Gamma(b)}{\Gamma(a+b)}$ is the Beta function, and $\Gamma(u)=$ $\int_{0}^{\infty} t^{u-1} e^{-t} d t$. Observing a labeling answer $Y_{i j}=y$, we can calculate the posterior distribution by Bayes' rule:

$$
p\left(\vartheta_{i} \mid Y_{i j}=y\right) \propto \operatorname{Pr}\left(Y_{i j}=y \mid \vartheta_{i}\right) \cdot p\left(\vartheta_{i}\right)
$$

Due to fact that Beta distribution is the conjugate prior of Bernoulli distribution, the posterior will become $\operatorname{Beta}\left(a_{i}^{0}+\right.$ $\left.1, b_{i}^{0}\right)$ if $Y_{i j}=1$, and $\operatorname{Beta}\left(a_{i}^{0}, b_{i}^{0}+1\right)$ if $Y_{i j}=0$. Thus, if task $t_{i}$ receives $r_{i}^{0}$ labels of 0 , and $r_{i}^{1}$ labels of 1 , the posterior will become

$$
p\left(\vartheta_{i} \mid \mathbf{y}_{i}\right)=\operatorname{Beta}\left(a_{i}^{0}+r_{i}^{0}, b_{i}^{0}+r_{i}^{1}\right)
$$


When we have no prior knowledge about the task, we can simply set $a_{i}^{0}=b_{i}^{0}=1$, so that the prior is a uniform distribution. The true label $z_{i}$ is inferred in accordance with soft label $\vartheta_{i}$, which indicates that $\mathbb{E}\left[\vartheta_{i}\right] \geq 0.5$ implies $z_{i}=1$.

\section{Aggregating Labels from Diverse Workers}

In practice, crowd workers are diverse in the quality. Another parameter $\varrho_{j} \in[0,1]$ is introduced to capture the quality of worker $j$. We model the distribution of $Y_{i j}$ by adopting the one-coin model

$$
\begin{aligned}
& \operatorname{Pr}\left(Y_{i j}=1 \mid \vartheta_{i}, \varrho_{j}\right)=\vartheta_{i} \varrho_{j}+\left(1-\vartheta_{i}\right)\left(1-\varrho_{j}\right) \\
& \operatorname{Pr}\left(Y_{i j}=0 \mid \vartheta_{i}, \varrho_{j}\right)=\vartheta_{i}\left(1-\varrho_{j}\right)+\left(1-\vartheta_{i}\right) \varrho_{j}
\end{aligned}
$$

As $\varrho_{j}$ increases, the quality of worker $j$ also increases in the sense that the distribution of $Y_{i j}$ gets closer to the underlying distribution of $\vartheta_{i}$. When $\varrho_{j}=1$, worker $j$ becomes a perfectly reliable worker.

Similarly, the quality $\varrho_{j}$ is drawn from a known Beta prior distribution $\operatorname{Beta}\left(c_{i}^{0}, d_{i}^{0}\right)$ [12]. Assuming that $\theta_{i}$ and $\varrho_{j}$ are independent, the prior joint distribution of is the product of two Beta distributions. Given a labeling answer from the crowd, the posterior distribution can be calculated by Bayes' rule:

$$
p\left(\vartheta_{i}, \varrho_{j} \mid Y_{i j}=y\right) \propto \operatorname{Pr}\left(Y_{i j}=y \mid \vartheta_{i}, \varrho_{j}\right) p\left(\vartheta_{i}, \varrho_{j}\right)
$$

In the posterior distribution, $\vartheta_{i}$ and $\varrho_{j}$ are highly correlated with a joint distribution and no longer follow the form of the product of two Beta distributions. We approximate the posterior distribution as follows:

$$
p\left(\vartheta_{i}, \varrho_{j} \mid Y_{i j}=y\right) \approx \tilde{p}\left(\vartheta_{i} \mid Y_{i j}=y\right) \tilde{p}\left(\varrho_{j} \mid Y_{i j}=y\right)
$$

where $\tilde{p}\left(\vartheta_{i} \mid Y_{i j}=y\right)$ and $\tilde{p}\left(\varrho_{j} \mid Y_{i j}=y\right)$ are all Beta distributions with modified parameters. By the approximation method in [12], the posterior distributions of $\vartheta_{i}$ and $\varrho_{j}$ are still two independent Beta distributions. Thus we can adopt the sequential allocation method. The detail of the approximation is revealed in the next section.

\section{Problem Formulation}

Suppose by the end of task allocation, each task $t_{i}$ receives labeling answers which can be represented as a vector $\mathbf{y}_{i}$ from the crowd and the posterior distributions of $\vartheta_{i}$ becomes $p\left(\vartheta_{i} \mid \mathbf{y}_{i}\right)$. Intuitively, if the posterior distribution is very different from the prior, our knowledge of the soft label is largely improved. We use the Kullback-Leibler divergence to measure the difference of two probability distributions and define the platform utility as follows.

Definition 2(PlatForm Utility). Platform utility is defined as the Kullback-Leibler divergence between the initial distribution and the final distribution of the soft labels.

$$
u_{p}\left(\Theta_{\mathcal{W}}\right)=\sum_{i=1}^{N} \operatorname{KL}\left(p\left(\vartheta_{i}\right)|| p\left(\vartheta_{i} \mid \mathbf{y}_{i}\right)\right)
$$

Under a strict budget constraint $B$, the platform aims to determine a winning bid set that maximizes its utility in expectation, i.e.

$$
\text { Maximize } \mathbb{E}\left[u_{p}\left(\Theta_{\mathcal{W}}\right)\right] \text { s.t. } \sum_{\theta \in \Theta_{\mathcal{W}}} p(\theta) \leq B
$$

If the platform pays exactly the price workers claim, $p\left(\theta_{j}^{k}\right)=b_{j}^{k}$, to every winning bid, the maximization problem can be formulated as a Markov Decision Process (MDP), which is described by a tuple $\left\{B^{r}, \Theta^{r}, \mathcal{S}^{r}, \operatorname{Pr}\left(Y_{i j} \mid S^{r}\right)\right\}$. Here, at current allocation round $r, B^{r}$ is the remaining budget. The action space $\Theta^{r}$ is all the remaining bids whose cost doesn't exceed the remaining budget. The state space $\mathcal{S}^{r}$ is all possible posterior parameters of soft labels, and quality parameters if workers are diverse. The transition probability $\operatorname{Pr}\left(Y_{i j} \mid S^{r}\right)$ has been described in our system model.

At each allocation round $r$, the platform can choose a $\theta^{r}$ from $\Theta^{r}$ as a winning bid and cover its cost with remaining budget. If $\theta^{r}$ is submitted by worker $j$ on task $t_{i}$, the expected reward of $\theta^{r}$ is

$$
R\left(\theta^{r} \mid S^{r}\right)=\mathbb{E}_{Y_{i j} \mid S^{r}}\left[u_{p}^{r+1}-u_{p}^{r}\right]
$$

where $u_{p}^{r}$ is the platform utility at round $r$. With the MDP in place, we can apply backward induction [14] to compute the optimal winning bid allocation scheme. However, this solution has two problems: 1) Since the cardinality of the state space $\left|\mathcal{S}^{r}\right|$ grows exponentially with the number of submitted bids, the optimal solution via backward induction is intractable. 2) Due to selfishness, workers strategically determine their claimed price to maximize his own utility, hindering the platform from hiring wisdom with lower payments.

Thus, our mechanism should be computationally efficient and truthful.

Definition 3(Computational Efficiency). A mechanism is computationally efficient if both the winning bid allocation algorithm and the payment determination algorithm terminate in polynomial time.

Definition 4(Truthfulness). Let $\tilde{\theta}_{j}^{k}=\left(t_{j}^{k}, c\left(t_{j}^{k}\right)\right)$ denote the truthful bid and $\theta_{j}^{k}=\left(t_{j}^{k}, b_{j}^{k}\right)$ denote the untruthful bid, where $b_{j}^{k} \neq c\left(t_{j}^{k}\right)$. The utility of the truthful bid and the untruthful bid is $u\left(\tilde{\theta}_{j}^{k}\right)=p\left(\tilde{\theta}_{j}^{k}\right)-c\left(t_{j}^{k}\right)$ and $u\left(\theta_{j}^{k}\right)=p\left(\theta_{j}^{k}\right)-c\left(t_{j}^{k}\right)$, respectively. The mechanism is truthful if

$$
u\left(\tilde{\theta}_{j}^{k}\right) \geq u\left(\theta_{j}^{k}\right)
$$

In order to maximize his utility, a worker will disclose the cost truthfully. Besides, the mechanism should also have the following desired properties.

Definition 5(Individual Rationality). The utility of a winning bid is nonnegative.

$$
p\left(\theta_{j}^{k}\right) \geq c\left(t_{j}^{k}\right)
$$


Definition 6(Budget Feasibility). The total payment to winning bids is less than the budget.

$$
\sum_{\theta \in \Theta_{\mathcal{W}}} p(\theta) \leq B
$$

Remarks: The importance of above two properties is obvious. To stimulate crowd workers to participate in task labeling, their costs must be covered by the payments. Budget feasibility guarantee that the mechanism can be implemented in practice and satisfy the basic requirement.

\section{Allocation And Payment Scheme}

\section{A. Allocation Scheme for Perfect Workers}

We present our winning bid allocation UN-GREEDY for perfect workers in this subsection. The allocation scheme is adaptive, i.e. the platform selects a winning bid, collects the labeling answer $Y_{i j}=y$, and update the posterior distribution of $\vartheta_{i}$ before choosing the next one.

Suppose in the current allocation round $r$, the distribution of $\vartheta_{i}$ is $p^{r}\left(\vartheta_{i}\right)=\operatorname{Beta}\left(a_{i}^{r}, b_{i}^{r}\right)$. By choosing a bid $\theta_{j}^{k}=\left(t_{j}^{k}=\right.$ $\left.t_{i}, b_{j}^{k}\right)$ and collecting answers $Y_{i j}=y$, the distribution of $\vartheta_{i}$ is updated as $p^{r+1}\left(\vartheta_{i}\right)=\operatorname{Beta}\left(a_{i}^{r+1}, b_{i}^{r+1}\right)$. The marginal value of $\theta_{j}^{k}$ at round $r$ is defined as the K-L divergence between $p^{r}\left(\vartheta_{i}\right)$ and $p^{r+1}\left(\vartheta_{i}\right)$ in expectation.

$$
v\left(\theta_{j}^{k} ; r\right)=\mathbb{E}_{Y_{i j}}\left[\operatorname{KL}\left(p^{r}\left(\vartheta_{i}\right) \| p^{r+1}\left(\vartheta_{i}\right)\right)\right]
$$

Once $\theta_{j}^{k}$ is selected into $\Theta_{\mathcal{W}}, v\left(\theta_{j}^{k}\right)$ is used instead of $v\left(\theta_{j}^{k} ; r\right)$ to simplify notations. The total value of $\Theta_{\mathcal{W}}$ is simply the sum of all marginal contributions, i.e.

$$
V\left(\Theta_{\mathcal{W}}\right)=\sum_{\theta_{j}^{k} \in \Theta_{\mathcal{W}}} v\left(\theta_{j}^{k}\right)
$$

Instead of measuring expected increment in platform utility, the marginal value is the myopic K-L difference between two successive allocation rounds. With this definition in place, the set function $V\left(\Theta_{\mathcal{W}}\right)$ falls in the family of monotone submodular functions.

Definition 7(Monotone Submodular Function). Let $\Omega$ be $a$ finite set. For any $X \subseteq Y \subseteq \Omega$ and $x \in \Omega / Y$, a function: $f: 2^{\Omega} \mapsto \mathbb{R}$ is submodular if and only if

$$
f(X \cup\{x\})-f(X) \geq f(Y \cup\{x\})-f(Y),
$$

and it is monotonic if and only if $f(X) \leq f(Y)$.

Theorem 1. The value function of the winning bid set is monotone submodular.

Proof: The value function is monotonic since the K$\mathrm{L}$ divergence is always positive. We only need to show the marginal value $v\left(\theta_{j}^{k} ; r\right)$ is monotonic decreasing with $r$ to prove the submodularity. The marginal value is a expectation taken over the answer $Y_{i j}$, which follows Bernoulli distribution that can be calculated as follows.

$$
\operatorname{Pr}\left(Y_{i j}=1\right)=\mathbb{E}_{\vartheta_{i}}\left(Y_{i j}=1 \mid \vartheta_{i}\right)=\mathbb{E}\left(\vartheta_{i}\right)=\frac{a_{i}^{r}}{a_{i}^{r}+b_{i}^{r}}
$$

$$
\operatorname{Pr}\left(Y_{i j}=0\right)=1-\operatorname{Pr}\left(Y_{i j}=1\right)=\frac{b_{i}^{r}}{a_{i}^{r}+b_{i}^{r}}
$$

The remainder of the proof is left over in the Appendix.

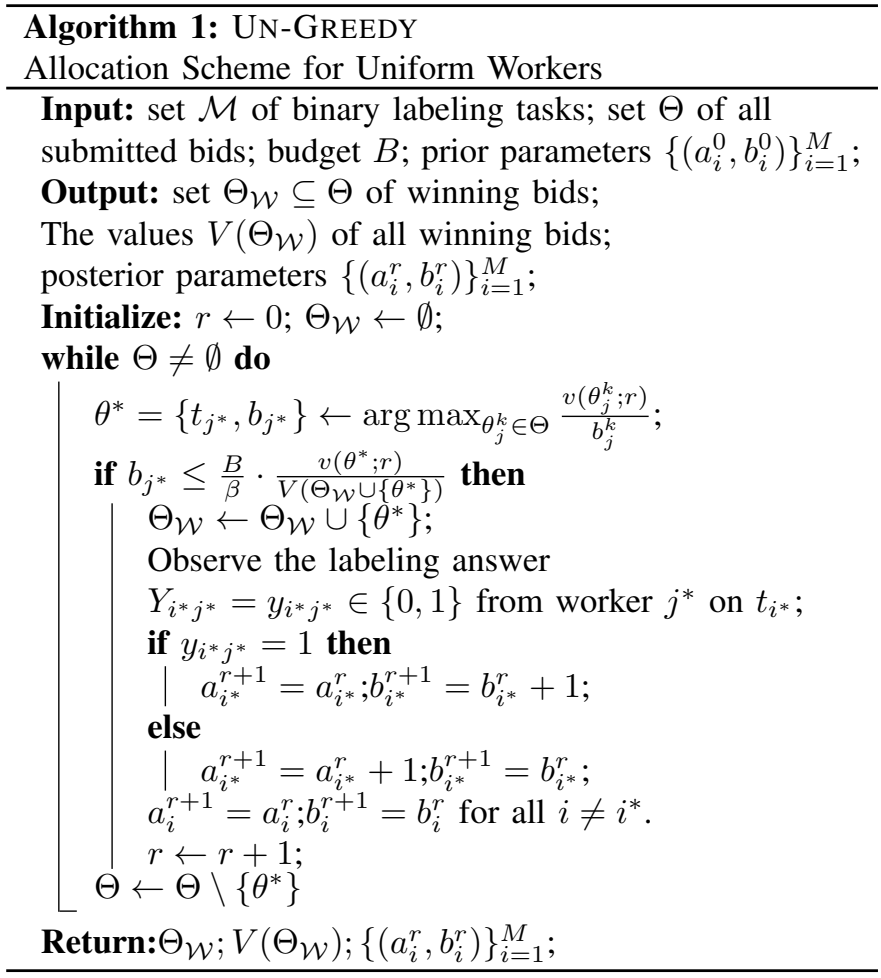

With this Lemma in place, we adapt the greedy allocation policy in [15] to present our winning bid selection method in Algorithm 1. In each allocation round $r$, the algorithm greedily selects the bid $\theta^{*}$ that has the largest value per unit cost, i.e.

$$
\theta^{*}=\left\{t_{j^{*}}, b_{j^{*}}\right\} \leftarrow \underset{\theta_{j}^{k} \in \Theta}{\arg \max } \frac{v\left(\theta_{j}^{k} ; r\right)}{b_{j}^{k}}
$$

Then we check if the candidate $\theta^{*}$ satisfies the proportional share rule on a reduced budget $B / \beta, \beta \geq 1$ to ensure budget feasibility, i.e.

$$
b_{j^{*}} \leq \frac{B}{\beta} \cdot \frac{v\left(\theta^{*} ; r\right)}{V\left(\Theta_{\mathcal{W}} \cup\left\{\theta^{*}\right\}\right)}
$$

If it holds, $\theta^{*}$ wins and the platform acquires the labeling answer to update posterior distributions. If the the candidate $\theta^{*}$ doesn't satisfy the proportional share rule, we simply discard it and the allocation goes into the next round.

The choice of $\beta_{\geq 1}$ will be discussed as part of the proof of budget feasibility.

\section{B. Allocation Scheme for Diverse Workers}

When taking the quality of crowd workers into consideration, the allocation scheme is almost the same except that the distribution parameters of $\vartheta_{i}, \varrho_{j}$ are updated by the approximation method. With the prior distributions of $\vartheta_{i}, \varrho_{j}$ being $\operatorname{Beta}\left(a_{i}, b_{i}\right), \operatorname{Beta}\left(c_{j}, d_{j}\right)$, respectively, the joint posterior distribution conditioned on the observed label $Y_{i j}=y$ is 
approximated as the product of two independent Beta distributions with modified parameters [12],

$$
\begin{aligned}
& \tilde{p}\left(\vartheta_{i} \mid Y_{i j}=y\right)=\operatorname{Beta}\left(a_{i}(y), b_{i}(y)\right) \\
& \tilde{p}\left(\varrho_{j} \mid Y_{i j}=y\right)=\operatorname{Beta}\left(c_{j}(y), d_{j}(y)\right)
\end{aligned}
$$

The values of $a_{i}(y), b_{i}(y)$, and $c_{j}(y), d_{j}(y)$ are calculated by moment matching, by setting the first and second moments of $\vartheta_{i}, \varrho_{j}$ equal in the true posterior $p\left(\vartheta_{i}, \varrho_{j} \mid Y_{i j}=y\right)$ and its approximation $\tilde{p}\left(\vartheta_{i} \mid Y_{i j}=y\right) \tilde{p}\left(\varrho_{j} \mid Y_{i j}=y\right)$. The details of calculation is in the appendix.

We present the winning bid allocation scheme for heterogeneous workers in Algorithm 2.

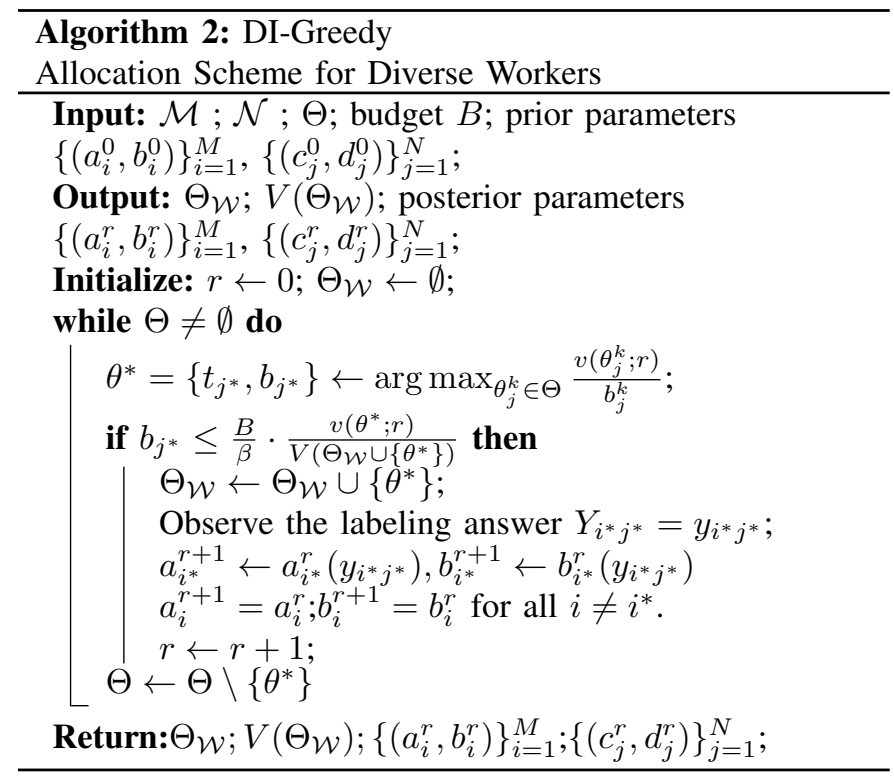

\section{Payment Scheme}

The payment scheme should guarantee truthfulness such that each worker reveals his true cost in the bid to maximize his utility, i.e. $b_{j}^{k}=c\left(t_{j}^{k}\right)$. Each winning bid is paid its threshold payment, which is equal to the highest price that still makes the bid win. Therefore the payment scheme for perfect and diverse workers can be unified. We adapt the payment scheme in [13] and the intuition behind can be described as follows. Let us number the winning bids as $\Theta_{\mathcal{W}}=\left\{\theta_{1}, \theta_{2}, \ldots, \theta_{i}, \ldots, \theta_{k}\right\}$ in the order of their selection when running the algorithm on the original bid set $\Theta$. We now consider the payment to the $i$-th allocated bid, $p\left(\theta_{i}\right)$. Running the allocation algorithm on the alternate set $\Theta^{\prime}=\Theta \backslash\left\{\theta_{i}\right\}$, we get an alternate allocation set $\Theta_{\mathcal{W}^{\prime}}=\left\{\theta_{1}^{\prime}, \theta_{2}^{\prime}, \ldots, \theta_{j}^{\prime}, \ldots, \theta_{k^{\prime}}^{\prime}\right\}$, in the order of their selection. To avoid confusion, we use $b\left(\theta_{j}^{\prime}\right)$ to denote the price that $\theta_{j}^{\prime} \in \Theta_{\mathcal{W}^{\prime}}$ declares. Now $\theta_{i}$ from $\Theta_{\mathcal{W}}$ wants to make a bid $b_{i(j)}$ to replace $\theta_{j}^{\prime}$ in $\Theta_{\mathcal{W}^{\prime}}$. This bid should be low enough to make $\theta_{j}$ 's value per cost larger than that of $\theta_{j}^{\prime}$, i.e.

$$
b_{i(j)} \leq \eta_{i(j)}=\frac{b\left(\theta_{j}^{\prime}\right) \cdot V_{i(j)}}{V\left(\theta_{j}^{\prime}\right)},
$$

where $v_{i(j)}$ is the marginal value of $\theta_{j}$ if it has to replace $\theta_{j}^{\prime}$ in $\Theta_{\mathcal{W}^{\prime}}$. Moreover, $b_{i(j)}$ should be low enough to follow the proportional share rule, i.e.

$$
b_{i(j)} \leq \rho_{i(j)}=\frac{B}{\beta} \cdot \frac{v_{i(j)}}{V\left(\Theta_{\mathcal{W}^{\prime}}^{j-1} \cup \theta_{i}\right)},
$$

where $\Theta_{\mathcal{W}^{\prime}}^{j-1}$ denotes the first $j-1$ elements in $\Theta_{\mathcal{W}^{\prime}}$.

Hence, the maximum bid that $\theta_{j}$ can declare to replace $\theta_{j}^{\prime}$ is $b_{i(j)}=\min \left(\eta_{i(j)}, \rho_{i(j)}\right)$. The final payment to $\theta_{i}$ takes the maximum value over the possible $k^{\prime}+1$ positions in $\Theta_{\mathcal{W}^{\prime}}$, $p\left(\theta_{i}\right)=\max _{j \in\left[k^{\prime}+1\right]} b_{i(j)}$.

In the next section, we will show that this is exactly the threshold payment of $\theta_{i}$ in part of the proof of truthfulness.

\section{Mechanism Analysis}

\section{A. Truthfulness}

According to the well-known statement by Myerson [16], a mechanism is truthful if and only if 1) the winning bid allocation is monotonic, 2) each winning bid is paid the threshold payment. We will show our mechanism satisfies these two conditions to finish the proof of truthfulness.

Lemma 1. If a bid $\theta_{j}^{k}=\left(t_{j}^{k}, b_{j}^{k}\right)$ wins, then the alternate bid $\tilde{\theta}_{j}^{k}=\left(t_{j}^{k}, \tilde{b}_{j}^{k}\right)$ also wins, $\tilde{b}_{j}^{k} \leq b_{j}^{k}$.

Proof: Let's consider the following two cases after $\theta_{j}^{k}$ lowers its price:

Case 1: By lowering $b_{j}^{k}$ to $\tilde{b}_{j}^{k}, \tilde{\theta}_{j}^{k}$ is chosen as the candidate in the same allocation round. Thus, we have $v\left(\theta_{j}^{k}\right)=v\left(\tilde{\theta}_{j}^{k}\right)$, and $V\left(\Theta_{\mathcal{W}} \cup\left\{\tilde{\theta}_{j}^{k}\right\}\right)=V\left(\Theta_{\mathcal{W}} \cup\left\{\theta_{j}^{k}\right\}\right)$. In this case, $\tilde{\theta}_{j}^{k}$ satisfies proportional share rule.

Case 2: $\tilde{\theta}_{j}^{k}$ is chosen as the candidate before $\theta_{j}^{k}$ is chosen. In this case, $\tilde{\theta}_{j}^{k}$ satisfies the proportional share rule due to the monotone submodularity of the value function.

$$
\tilde{b}_{j}^{k}<b_{j}^{k} \leq \frac{B}{\beta} \cdot \frac{v\left(\theta_{j}^{k}\right)}{V\left(\Theta_{\mathcal{W}} \cup\left\{\theta_{j}^{k}\right\}\right)} \leq \frac{B}{\beta} \cdot \frac{v\left(\tilde{\theta}_{j}^{k}\right)}{V\left(\tilde{\Theta}_{\mathcal{W}} \cup\left\{\tilde{\theta}_{j}^{k}\right\}\right)}
$$

where $\tilde{\Theta}_{\mathcal{W}}$ denotes the winning bid set right before $\tilde{\theta}_{j}^{k}$ is chosen as the candidate.

Notice $\tilde{\theta}_{j}^{k}$ cannot be chosen after $\theta_{j}^{k}$ since our determination rule is greedy.

Lemma 2. Each winning bid $\theta_{i}=\left(t_{i}, b_{i}\right) \in \Theta_{\mathcal{W}}$ is paid its threshold payment, i.e. $p\left(\theta_{i}\right)=\inf \left\{b_{i}: \theta_{i} \notin \Theta_{\mathcal{W}}\right\}$

Proof: The payment to each winning bid in $\Theta_{\mathcal{W}}$ is given as $p\left(\theta_{j}\right)=\max _{j \in\left[k^{\prime}+1\right]} b_{i(j)}$, where $b_{i(j)}=\min \left(\eta_{i(j)}, \rho_{i(j)}\right)$.

Assume by replaces $m$-th winning bid in $\Theta_{\mathcal{W}^{\prime}}, \theta_{i}$ can declare the maximum bid, i.e $p\left(\theta_{j}\right)=b_{i(m)}$. We then adapt the proof in [15] to show that $\theta_{i}$ cannot win if his bid $b_{i}>b_{i(m)}$.

Let $\pi_{i(m)}=\eta_{i(m)} \leq \rho_{i(m)}$. If $\pi_{i(m)}=\max _{j \in\left[k^{\prime}+1\right]} \pi_{i(j)}$, Submitting a price higher than $\pi_{i(m)}$ places $\theta_{i}$ after the unallocated bid $\theta_{k^{\prime}+1}^{\prime}$ in $\Theta_{\mathcal{W}^{\prime}}$. Thus, $\theta_{i}$ cannot win. If $\pi_{i(m)}<$ $\max _{j \in\left[k^{\prime}+1\right]} \pi_{i(j)}$, consider some $j$ such that $\pi_{i(m)}<\pi_{i(j)}$. It is shown in [15] that submitting a price larger than $\pi_{i(m)}$ cannot 
win since it would violate either the proportional share rule or put $\theta_{i}$ after $\theta_{j}^{\prime}$.

\section{Theorem 2. The winning bid allocation is truthful.}

Proof: According to the well-known statement by Myerson [16], this theorem holds from the previous two Lemmas.

\section{B. Individual Rationality}

Theorem 3. Let $\theta_{i}=\left(t_{i}, b_{i}\right)$. The utility of a winning bid is nonnegative, i.e. $p\left(\theta_{i}\right) \geq c\left(t_{i}\right), \forall \theta_{i} \in \Theta_{\mathcal{W}}$.

Proof: Since $p\left(\theta_{i}\right)=\max _{j \in\left[k^{\prime}+1\right]} b_{i(j)}$, we only need to show that $b_{i(j)} \geq b_{i}$ for some $j \in\left[k^{\prime}+1\right]$, then the individual rationality holds due to the truthfulness, $b_{i}=c\left(t_{i}\right)$. We adapt the proof in the extended version of [15] to show the individual rationality. We observe that in the original winning bid set $\Theta_{\mathcal{W}}$ and the winning bid set $\Theta_{\mathcal{W}^{\prime}}$ without $\theta_{i}$ : 1) The first $i-1$ winning bids in the two sets are identical, $\Theta_{\mathcal{W}}^{i-1}=\Theta_{\mathcal{W}^{\prime}}^{i-1}$. 2) $\theta_{i}$ from $\Theta_{\mathcal{W}}$ can replace a winning bid from $\Theta_{\mathcal{W}}$ in the same position, by submitting $b_{i(i)}=\min \left(\eta_{i(i)}, \rho_{i(i)}\right)$.

According to observation 1) we have $v_{i(i)}=v\left(\theta_{i}\right)$. Then

$$
\eta_{i(i)}=\frac{b\left(\theta_{j}^{\prime}\right) \cdot v_{i(i)}}{v\left(\theta_{j}^{\prime}\right)}=v\left(\theta_{i}\right) \cdot \frac{b\left(\theta_{j}^{\prime}\right)}{v\left(\theta_{j}^{\prime}\right)} \geq v\left(\theta_{i}\right) \cdot \frac{b_{i}}{v\left(\theta_{i}\right)}=b_{i}
$$

and

$$
\rho_{i(i)}=\frac{B}{\beta} \cdot \frac{v_{i(i)}}{V\left(\Theta_{\mathcal{W}^{\prime}}^{i-1} \cup \theta_{i}\right)}=\frac{B}{\beta} \cdot \frac{v_{i}}{V\left(\Theta_{\mathcal{W}}^{i-1} \cup \theta_{i}\right)} \geq b_{i}
$$

Thus, we have $b_{i} \leq \min \left(\eta_{i(i)}, \rho_{i(i)}\right) \leq p\left(\theta_{i}\right)$.

\section{Budget Feasibility}

The allocation scheme is based on the proportional share rule that runs on a fraction of the total budget $B / \beta$, where $\beta_{\geq 1}$ is the budget fraction ratio. Intuitively, a larger $\beta$ better guarantees the budget constraint, while a smaller $\beta$ better utilizes the budget. A fraction ratio of 2 is achieved by Singla et al. in [15].

Assume the winning bid selection runs with full budget $B$ first, i.e. $\beta=1$, with $\Theta_{\mathcal{W}}=\left\{\theta_{1}, \theta_{2}, \ldots, \theta_{i}, \ldots, \theta_{k}\right\}$ being the winning bids. We will upper bound the payment $p\left(\theta_{i}\right) \in \Theta_{\mathcal{W}}$, by bounding the maximum raise $\theta_{i}$ can make in its bid $b_{i}$ and still wins. We formalize it in the next lemma.

Lemma 3. When full budget is used in the winning bid selection algorithm, the maximum bid $b_{i}^{\prime}$ that a winning bid can make and still wins is upper bounded by $2 \cdot \frac{v\left(\theta_{i}\right)}{V\left(\Theta_{\mathcal{W}}\right)} \cdot B$.

Proof: Suppose a winning bid $\theta_{i} \in \Theta_{\mathcal{W}}$ raises its bid from $b_{i}^{\prime}\left(>b_{i}\right)$, and still wins. Let $\Theta_{\mathcal{W}^{\prime}}=\left\{\theta_{1}^{\prime}, \ldots, \theta_{j}^{\prime}\left(=\theta_{i}\right), \ldots, \theta_{k^{\prime}}^{\prime}\right\}$ be the alternate winning set, where $\theta_{i}$ with raised price gets selected at the $j$-th position.

Since the bid is raised, $\theta_{i}$ in $\Theta_{\mathcal{W}^{\prime}}$ gets selected after in $\Theta_{\mathcal{W}}(j>i)$, or at least at the same position $(j=i)$. Let $\Theta_{\mathcal{W}}^{i}, \Theta_{\mathcal{W}}^{j}$ denote the first $i, j$ winning bids in $\Theta_{\mathcal{W}}, \Theta_{\mathcal{W}^{\prime}}$, respectively. As in the extended version of [15], we now investigate $\Theta_{\mathcal{R}}=\Theta_{\mathcal{W}} \backslash \Theta_{\mathcal{W}^{\prime}}^{j}$ in the following two cases.

Case 1: $\Theta_{\mathcal{R}}=\emptyset$.

This also implies that $\Theta_{\mathcal{W}} \subset \Theta_{\mathcal{W}^{\prime}}^{j}$, and $V\left(\Theta_{\mathcal{W}}\right)<V\left(\Theta_{\mathcal{W}^{\prime}}^{j}\right)$ according to the non-negativity of value function. Thus we have

$$
b_{i}^{\prime} \leq B \cdot \frac{v\left(\theta_{j}^{\prime}\right)}{V\left(\Theta_{\mathcal{W}^{\prime}}^{j}\right)} \leq B \cdot \frac{v\left(\theta_{j}^{\prime}\right)}{V\left(\Theta_{\mathcal{W}}\right)} \leq B \cdot \frac{v\left(\theta_{i}\right)}{V\left(\Theta_{\mathcal{W}}\right)}
$$

In the last inequality, $v\left(\theta_{j}^{\prime}\right) \leq v\left(\theta_{i}\right)$ holds due to the decreasing marginal value property. In this case, $b_{i}^{\prime}$ is strictly upper bounded by the proportional value of $\theta_{i}$.

Case 2: $\Theta_{\mathcal{R}} \neq \emptyset$.

Assume $b_{i}^{\prime}=\alpha \cdot B \frac{V\left(\theta_{i}\right)}{V\left(\Theta_{\mathcal{W}}\right)}$. Still, we have

$$
b_{i}^{\prime} \leq B \cdot \frac{v\left(\theta_{j}^{\prime}\right)}{V\left(\Theta_{\mathcal{W}^{\prime}}^{j}\right)} \leq B \cdot \frac{v\left(\theta_{i}\right)}{V\left(\Theta_{\mathcal{W}^{\prime}}^{j}\right)}
$$

Hence $\alpha \leq \frac{V\left(\Theta_{\mathcal{W}}\right)}{V\left(\Theta_{\mathcal{W}^{\prime}}^{j}\right)}$.

Now, consider replacing $\theta_{j}^{\prime}$ with some $\theta_{r_{0}} \in \Theta_{\mathcal{R}}$ after $\Theta_{\mathcal{W}^{\prime}}^{j-1}$. It must hold that the marginal value per cost of $\theta_{r_{0}}$ is higher than that of the whole $\Theta_{\mathcal{R}}$. To simplify notations, we use $v(\theta \mid \Theta)=\frac{V(\Theta \cup \theta)-V(\Theta)}{b(\theta)}$ to denote the marginal value of a bid $\theta$, if it is selected after a set of bids $\Theta$. We have

$$
\frac{V\left(\Theta_{\mathcal{W}^{\prime}}^{j} \cup \Theta_{\mathcal{R}}\right)-V\left(\Theta_{\mathcal{W}^{\prime}}^{j}\right)}{\sum_{\theta_{r} \in \Theta_{\mathcal{R}}} b\left(\theta_{r}\right)} \leq \frac{v\left(\theta_{r 0} \mid \Theta_{\mathcal{W}^{\prime}}^{j}\right)}{b\left(\theta_{r 0}\right)} \leq \frac{v\left(\theta_{r 0} \mid \Theta_{\mathcal{W}^{\prime}}^{j-1}\right)}{b\left(\theta_{r 0}\right)}
$$

The second inequality is due to the submodularity of the value function. Since $\theta_{j}^{\prime}$ is chosen as the candidate at position $j$ instead of $\theta_{r 0}$, we have

$$
\frac{v\left(\theta_{r 0} \mid \Theta_{\mathcal{W}^{\prime}}^{j-1}\right)}{b\left(\theta_{r 0}\right)} \leq \frac{v\left(\theta_{j}^{\prime} \mid \Theta_{\mathcal{W}^{\prime}}^{j-1}\right)}{b_{i}^{\prime}} \leq \frac{v\left(\theta_{i} \mid \Theta_{\mathcal{W}}^{i-1}\right)}{b_{i}^{\prime}}=\frac{V\left(\Theta_{\mathcal{W}}\right)}{\alpha \cdot B}
$$

Observing that $\sum_{\theta_{r} \in \Theta_{\mathcal{R}}} b\left(\theta_{r}\right) \leq B$, and $V\left(\Theta_{\mathcal{W}}\right) \leq$ $V\left(\Theta_{\mathcal{W}^{\prime}}^{j} \cup \Theta_{\mathcal{R}}\right)$, we have

$$
\frac{V\left(\Theta_{\mathcal{W}}\right)-V\left(\Theta_{\mathcal{W}^{\prime}}^{j}\right)}{B} \leq \frac{V\left(\Theta_{\mathcal{W}}\right)}{\alpha \cdot B}
$$

which leads to $\frac{V\left(\Theta_{\mathcal{W}}\right)}{V\left(\Theta_{\mathcal{W}^{\prime}}^{j}\right)} \leq \frac{\alpha}{\alpha-1}$. Combining the constraints on $\alpha$ together, we have $\alpha=2$.

Hence, $b_{i}^{\prime} \leq 2 \cdot \frac{v\left(\theta_{i}\right)}{V\left(\Theta_{\mathcal{W}}\right)} \cdot B$ holds in the above two cases.

With this lemma in place, the proof of the budget feasibility is straight forward.

Theorem 4. Our mechanism achieves budget feasibility, i.e. $\sum_{\theta_{i} \in \Theta_{\mathcal{W}}} p\left(\theta_{i}\right) \leq B$, by choosing the budget fraction ratio $\beta=2$.

Proof: Running the winning bid allocation algorithm with $B / 2$, we have

$$
\sum_{\theta_{i} \in \Theta_{\mathcal{W}}} b_{i}^{\prime} \leq \sum_{\theta_{i} \in \Theta_{\mathcal{W}}} 2 \cdot \frac{v\left(\theta_{i}\right)}{V\left(\Theta_{\mathcal{W}}\right)} \cdot \frac{B}{2}=B
$$


Since $p\left(\theta_{i}\right)$ is the threshold payment, i.e. $p\left(\theta_{i}\right)=\sup \left\{b_{i}^{\prime}\right.$ : $\left.\theta_{i} \in \Theta_{\mathcal{W}^{\prime}}\right\}$, by taking the supremum we have

$$
\sup \sum_{\theta_{i} \in \Theta_{\mathcal{W}}} b_{i}^{\prime}=\sum_{\theta_{i} \in \Theta_{\mathcal{W}}} p\left(\theta_{i}\right) \leq B
$$

\section{Computation Efficiency}

Theorem 5. Both winning bid allocation scheme and payment scheme are computationally efficient.

Proof: In winning bid allocation algorithm, the sorting before selecting the candidate bid $\theta^{*}$ is $O(|\Theta| \log |\Theta|)$. The proportional share rule checking and posterior parameter updating is $O(1)$. Since the outer while loop runs at most $|\Theta|$ times, the computation complexity for allocation algorithm is $O\left(|\Theta|^{2} \log |\Theta|\right)$.

The payment scheme first runs $\left|\Theta_{\mathcal{W}}\right|$ times for every winning bid $\theta_{i}$ on $\Theta \backslash\left\{\theta_{i}\right\}$, which is $O\left(\left|\Theta_{\mathcal{W}}\right| \cdot|\Theta|^{2} \log |\Theta|\right)$. Then it chooses the maximum value $b_{i(m)}$ over all the possible positions in $\Theta_{\mathcal{W}}^{\prime}$. Thus the payment scheme is $O\left(\left|\Theta_{\mathcal{W}}\right|\right.$. $\left.\left(|\Theta|^{2} \log |\Theta|+\left|\Theta_{\mathcal{W}}^{\prime}\right|\right)\right)$.

\section{Performance Evaluation}

To effectively evaluate the performance of our mechanism, we implement our winning bid allocation algorithms UNGREEDY and DI-GREEDY against the following benchmarks.

- UN-RANDOM regards all workers as uniform and perfect. It randomly chooses a bid outside the current winning bid set as the candidate. The candidate also needs to respect the proportional share rule before becoming a winning bid.

- Di-RANDOM is the equivalent to UN-RANDOM in the sense that it incorporates workers' quality levels to update posterior distributions.

- UN-UntRUthful has access to workers' real costs and pays the exact cost to the worker. It chooses the candidate the same way as UN-GREEDY. The candidate becomes a winning bid if its cost is less than the remaining budget.

- Di-Untruthful is the equivalent to Un-UntruthFul in the sense that it incorporates workers' quality.

The performance metrics includes running time, platform utility, and budget utilization. To evaluate the computation efficiency, we compare the running time of UN-GREEDY and DI-GREEDY against the benchmarks. We then evaluate platform utility to show that our mechanism outperforms the naive random allocation scheme. Finally, we compute the total payment to show that our mechanism utilizes budget efficiently under strict constraints.

\section{A. Running Time}

We set the cost of each bids as uniformly distributed over $[1,5]$, and vary the number of submitted bids $N$ from 100 to 600 for different allocation algorithms. The running time of uniform workers and diverse workers are shown in Fig. 1. We observe that the running time of UN-GREEDY and
DI-GREEDY is much less that UN-UNTRUTHFUL and DIUNTRUTHFUL, respectively. UN-RANDOM and DI-RANDOM outperform the rest since they doesn't do sorting operation. The running time of UN-GREEDY and DI-GREEDY increases lineally with the number of bids $N$, while the budget $B$ doesn't affect running time much.

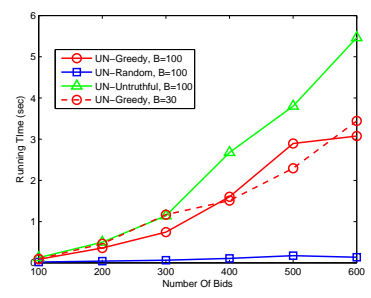

(a) Uniform workers

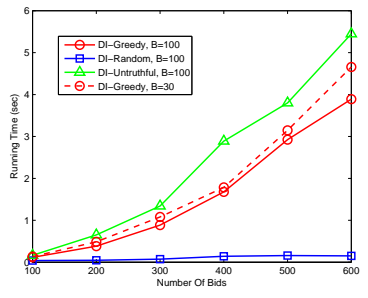

(b) Diverse workers
Fig. 1. Running time

\section{B. Platform Utility}

We first assume the cost is uniformly distributed over $[1,5]$ and vary the budget $B$. The platform utility of uniform workers under different winning bid allocation algorithms is plotted in Fig. 2(a). Fig. 2(b) plots the platform utility in the case of diverse workers. We observe that UN-UNTRUTHFUL outperforms UN-GREEDY slightly since it does not respect proportional share rule and pays exactly the cost to winning bids. When the number of bids $N=200$, UN-GrEEDY always outperforms UN-RANDOM for all budget constraints. As the number of bids $N$ reduces to 50, platform utility also decreases, which reveals that a large amount of bids brings more utility.

Next, we generate the costs of bids according to three distributions: uniform distribution (UNM), normal distribution (NORM) and exponential distribution (EXP) with equal mean value 5.5. The variance of the normal distribution is set as $\sigma^{2}=1$. The platform utility is plotted in Fig. 3 .

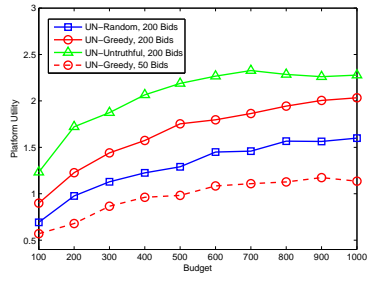

(a) Uniform workers

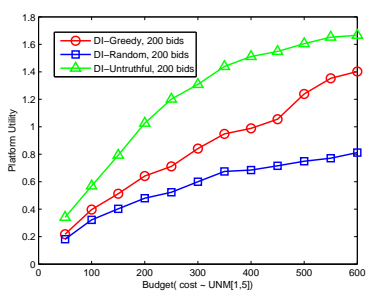

(b) Diverse workers
Fig. 2. Platform utility

\section{Budget Utilization}

We compute the total payment corresponding to UNGreEDY, Di-GreEDY, UN-RANDOM, and Di-RANDOM as budget $B$ ranging from 50 to 400 . The number of bids is set to 100 and the cost follows uniform distribution over $[1,5]$. We plot the budget utilization ratio $P / B$ in Fig. 4. The results 
show that our allocation algorithms utilize budget much more efficient than the random allocation scheme.

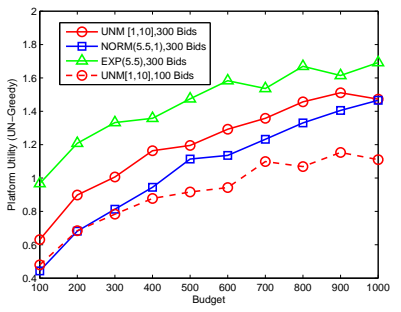

Fig. 3. Impact of cost

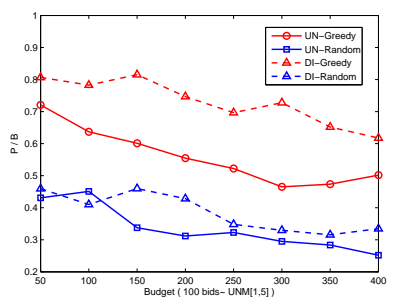

Fig. 4. Budget utilization

\section{RELATED WORK}

\section{A. Crowd Label Collection and Aggregation}

Much of the previous work focus on information aggregation in a crowdsourcing scenario where we have to infer the true label with a bunch of noisy labels. Karger et al. [8], [10] allocate labeling tasks according to a bipartite graph and infer the true label with an iterative learning algorithm, or low-rank matrix approximation. Raykar et al. [17], [11] propose a probabilistic framework with multiple annotators providing labels but no absolute gold standard. Xiao et al. [18] use the BCH code for multi-class labeling in the setting of mobile crowdsensing. Sheng et al. [19] consider inference methods for crowdsourcing based scoring systems. However, these papers assume that we can repeatedly access any worker for any tasks and fail to provide appropriate incentives.

\section{B. Incentive Mechanisms for Crowd Sensing}

Most existing incentive mechanisms focus on the system of mobile sensing applications. Yang et al. [9] design two incentive mechanisms for a user centric model and a platform centric model, respectively. He et al. [20], Feng et al. [21] and James et al. [22] study the task allocation problem for location dependent tasks. Iosifidis et al. [23] design a bargaining based scheme for the crowdsourced Internet access. Lee et al. [24] design a reverse auction based mechanism with dynamic price. Zhang et al. [25] design a non-monetary incentive mechanism based on participants' reputation. Zhao et al. [26] design an online mechanism for mobile crowdsourced sensing. However, there are few works that adapt the desired properties such as truthfulness and budget feasibility into the setting of crowdsourced label collection.

\section{CONCLUSions And Future Work}

In this paper, we have presented a framework of reverse auction based incentive mechanism for label collection in crowdsourcing systems. We take into account the difficulty of labeling tasks and focus on maximizing the utility of the crowdsourcer. We have designed two winning bid allocation algorithm under different assumptions: UN-GREEDY can be applied to the case where the majority of crowd workers are of high reliability, and DI-GREEDY can be applied to a more general case where the workers are highly diverse. The proposed mechanism is easy to implement in practice, and has strong theoretical guarantees such as truthfulness, individual rationality, budget feasibility and computation efficiency.

We can carry the future work along the following directions. First, the tasks can be extended to multi-class labeling, in which a task may get more than two labels. Second, since the workers may arrive and leave at any time online, we can extend our mechanism to this highly dynamic scenario.

\section{ACKNOWLEDGEMENT}

This work is supported by NSF China (No.61325012, 61271219, 61221001, 61202373, 61102052, 61102051, 61428205); China Ministry of Education Doctor Program (No.20130073110025); SEU SKL project (No.2012D13, No. 2014D07); Shanghai Basic Research Key Project (No. 13510711300, 12JC1405200, 11JC1405100); Shanghai International Cooperation Project: (No. 13510711300); Jiangsu Future Network Research Project: (No. BY2013095-1-10).

\section{REFERENCES}

[1] L. Von Ahn, B. Maurer, C. McMillen, D. Abraham, and M. Blum, "recaptcha: Human-based character recognition via web security measures," Science, vol. 321, no. 5895, pp. 1465-1468, 2008.

[2] L. von Ahn, "Games with a purpose," IEEE Computer, vol. 39, no. 6, pp. 92-94, 2006.

[3] S. Jain, Y. Chen, and D. C. Parkes, "Designing incentives for online question and answer forums," in Proceedings of the 10th ACM conference on Electronic commerce. ACM, 2009, pp. 129-138.

[4] Amazon mturk, http://www.mturk.com.

[5] Clickworker, http://www.clickworker.com.

[6] CloudCrowd, http://www.cloudcrowd.com.

[7] Crowdflower, http://www.crowdflower.com.

[8] D. R. Karger, S. Oh, and D. Shah, "Iterative learning for reliable crowdsourcing systems," in Advances in neural information processing systems, 2011, pp. 1953-1961.

[9] D. Yang, G. Xue, X. Fang, and J. Tang, "Crowdsourcing to smartphones: incentive mechanism design for mobile phone sensing," in Proceedings of the 18th annual international conference on Mobile computing and networking. ACM, 2012, pp. 173-184.

[10] D. R. Karger, S. Oh, and D. Shah, "Efficient crowdsourcing for multiclass labeling," in Proceedings of the ACM SIGMETRICS/international conference on Measurement and modeling of computer systems. ACM, 2013, pp. 81-92.

[11] V. C. Raykar and S. Yu, "Ranking annotators for crowdsourced labeling tasks," in Advances in neural information processing systems, 2011, pp. 1809-1817.

[12] X. Chen, Q. Lin, and D. Zhou, "Optimistic knowledge gradient policy for optimal budget allocation in crowdsourcing," in Proceedings of the 30th International Conference on Machine Learning (ICML-13), 2013, pp. 64-72.

[13] Y. Singer, "Budget feasible mechanisms," in Foundations of Computer Science (FOCS), 2010 51st Annual IEEE Symposium on, pp. 765-774.

[14] R. J. Aumann, "Backward induction and common knowledge of rationality," Games and Economic Behavior, vol. 8, no. 1, pp. 6-19, 1995.

[15] A. Singla and A. Krause, "Incentives for privacy tradeoff in community sensing," in First AAAI Conference on Human Computation and Crowdsourcing, 2013.

[16] R. B. Myerson, "Optimal auction design," Mathematics of operations research, vol. 6, no. 1, pp. 58-73, 1981.

[17] V. C. Raykar, S. Yu, L. H. Zhao, G. H. Valadez, C. Florin, L. Bogoni, and L. Moy, "Learning from crowds," The Journal of Machine Learning Research, vol. 11, pp. 1297-1322, 2010.

[18] X. Xiao, X. Tian, X. Gan, and X. Wang, "Multi-class labeling with bch codes for mobile crowdsensing," in Global Communications Conference (GLOBECOM), 2014 IEEE.

[19] K. Sheng, Z. Gu, X. Mao, X. Tian, X. Gan, and X. Wang, "Answer inference for crowdsourcing based scoring," in Global Communications Conference (GLOBECOM), 2014 IEEE. 
[20] S. He, D.-H. Shin, J. Zhang, and J. Chen, "Toward optimal allocation of location dependent tasks in crowdsensing," in INFOCOM, 2014 Proceedings IEEE.

[21] Z. Feng, Y. Zhu, Q. Zhang, L. M. Ni, and A. V. Vasilakos, "Trac: Truthful auction for location-aware collaborative sensing in mobile crowdsourcing," in INFOCOM, 2014 Proceedings IEEE, pp. 1231-1239.

[22] L. G. Jaimes, I. Vergara-Laurens, and M. A. Labrador, "A location-based incentive mechanism for participatory sensing systems with budget constraints," in Pervasive Computing and Communications (PerCom), 2012 IEEE International Conference on, pp. 103-108.

[23] G. Iosifidis, L. Gao, J. Huang, and L. Tassiulas, "Enabling crowdsourced mobile internet access," in INFOCOM, 2014 Proceedings IEEE, pp. 451-459.

[24] J.-S. Lee and B. Hoh, "Sell your experiences: a market mechanism based incentive for participatory sensing," in Pervasive Computing and Communications (PerCom), 2010 IEEE International Conference on, pp. 60-68.

[25] Y. Zhang and M. van der Schaar, "Reputation-based incentive protocols in crowdsourcing applications," in INFOCOM, 2012 Proceedings IEEE, pp. $2140-2148$

[26] D. Zhao, X.-Y. Li, and H. Ma, "How to crowdsource tasks truthfully without sacrificing utility: Online incentive mechanisms with budget constraint," in INFOCOM, 2014 Proceedings IEEE, pp. 1213-1221.

[27] M. Abramowitz and I. A. Stegun, Handbook of mathematical functions: with formulas, graphs, and mathematical tables. Courier Dover Publications, 1972, no. 55.

\section{APPENDIX A}

\section{PROOF OF SUBMODULARITY}

The K-L divergence of two Beta distributions is

$$
\begin{aligned}
& \mathrm{KL}\left(\operatorname{Beta}(a, b) \| \operatorname{Beta}\left(a^{\prime}, b^{\prime}\right)\right)=\ln \left(\frac{\mathrm{B}\left(a^{\prime}, b^{\prime}\right)}{\mathrm{B}(a, b)}\right) \\
& +\left(a-a^{\prime}\right) \psi(a)+\left(b-b^{\prime}\right) \psi(b)+\left(a^{\prime}-a+b^{\prime}-b\right) \psi(a+b)
\end{aligned}
$$

where $\psi(x)=\frac{\Gamma^{\prime}(x)}{\Gamma(x)}$ is the digamma function. In the setting of uniform workers, the marginal value of a bid $\theta_{j}^{k}$ at round $r$ can be calculated as

$$
\begin{aligned}
v\left(\theta_{j}^{k} ; r\right) & =p_{0} \cdot \operatorname{KL}\left(\operatorname{Beta}\left(a_{i}^{r}, b_{i}^{r}\right) \| \operatorname{Beta}\left(a_{i}^{r}, b_{i}^{r}+1\right)\right) \\
& +p_{1} \cdot \operatorname{KL}\left(\operatorname{Beta}\left(a_{i}^{r}, b_{i}^{r}\right) \| \operatorname{Beta}\left(a_{i}^{r}+1, b_{i}^{r}\right)\right)
\end{aligned}
$$

where $a_{i}^{r}, b_{i}^{r}$ are posterior parameters for task $t_{i}$ in round $r$. Since $\mathrm{B}(a+1, b)=\frac{a}{a+b} \mathrm{~B}(a, b)$, the above formula is defined as $v\left(a_{i}^{r}, b_{i}^{r}\right)$ and can be simplified into

$$
\begin{aligned}
v\left(a_{i}^{r}, b_{i}^{r}\right) & =\psi\left(a_{i}^{r}+b_{i}^{r}\right)-\ln \left(a_{i}^{r}+b_{i}^{r}\right) \\
& +\frac{a_{i}^{r}}{a_{i}^{r}+b_{i}^{r}}\left[\ln \left(a_{i}^{r}\right)-\psi\left(a_{i}^{r}\right)\right]+\frac{b_{i}^{r}}{a_{i}^{r}+b_{i}^{r}}\left[\ln \left(b_{i}^{r}\right)-\psi\left(b_{i}^{r}\right)\right]
\end{aligned}
$$

To prove $v\left(\theta_{j}^{k} ; r\right)$ is monotonic decreasing with $r$, we shall show that $v\left(a^{\prime}, b^{\prime}\right) \leq v(a, b)$, for $a^{\prime} \geq a, b^{\prime} \geq b$. Since $a_{i}^{r}$ and $b_{i}^{r}$ are symmetric, we only need to show that $\frac{\partial v(a, b)}{\partial a} \leq 0$.

$$
\begin{aligned}
\frac{\partial v(a, b)}{\partial a} & =\psi^{(1)}(a+b)-\frac{a}{a+b} \psi^{(1)}(a) \\
& +\frac{b}{(a+b)^{2}}[\ln (a)-\ln (b)+\psi(b)-\psi(a)]
\end{aligned}
$$

where $\psi^{(m)}(z)=(-1)^{m+1} m ! \sum_{k=0}^{\infty} \frac{1}{(z+k)^{m+1}}$, for $m>0$ and any complex $z$ not equal to a negative integer [27]. We have

$$
\frac{d}{d x}\left(\psi^{(1)}(x)-\frac{1}{x-0.49}\right)=\sum_{k=0}^{\infty} \frac{-2}{(x+k)^{3}}+\frac{1}{(x-0.49)^{2}} \leq 0,
$$

Since $\psi^{(1)}(x) \geq \psi(x+1)-\psi(x)=\frac{1}{x}$, we get

$$
\lim _{x \rightarrow \infty} \psi^{(1)}(x)-\frac{1}{x-0.49}=0
$$

Thus,

$$
\psi^{(1)}(x) \geq \frac{1}{x-0.49}
$$

We get another inequality from

$$
\psi^{(1)}(x) \leq \psi(x+0.5)-\psi(x-0.5)=\frac{1}{x-0.5}
$$

Submitting 35, 36 into 32 ends the proof.

\section{APPENDIX B \\ Posterior CALCUlation}

Assuming, at the current state, $\vartheta_{i}$ and $\varrho_{j}$ are independent, and $\vartheta_{i} \sim \operatorname{Beta}\left(a_{i}, b_{i}\right), \varrho_{j} \sim \operatorname{Beta}\left(c_{j}, d_{j}\right)$, the posterior distribution conditioned on $Y_{i j}$ can be calculated using Bayes' rule:

$$
p\left(\vartheta_{i}, \varrho_{j} \mid Y_{i j}=y\right)=\frac{\operatorname{Pr}\left(Y_{i j}=y \mid \vartheta_{i}, \varrho_{j}\right) p\left(\vartheta_{i}, \varrho_{j}\right)}{\operatorname{Pr}\left(Y_{i j}=y\right)}
$$

for $y=1,0$, where

$$
\begin{gathered}
p\left(\vartheta_{i}, \varrho_{j}\right)=p\left(\vartheta_{i}\right) p\left(\varrho_{j}\right)=\operatorname{Beta}\left(a_{i}, b_{i}\right) \cdot \operatorname{Beta}\left(c_{j}, d_{j}\right) \\
\operatorname{Pr}\left(Y_{i j}=y\right)=\mathbb{E}_{\vartheta_{i}, \varrho_{j}}\left(\operatorname{Pr}\left(Y_{i j}=y \mid \vartheta_{i}, \varrho_{j}\right)\right)
\end{gathered}
$$

We approximate the posterior distribution by a product of two Beta distribution with modified parameters as done by Chen et al. in [12],

$$
\begin{aligned}
p\left(\vartheta_{i}, \varrho_{j} \mid Y_{i j}=y\right) & \approx \tilde{p}\left(\vartheta_{i} \mid Y_{i j}=y\right) \tilde{p}\left(\varrho_{j} \mid Y_{i j}=y\right) \\
& =\operatorname{Beta}\left(a_{i}(y), b_{i}(y)\right) \operatorname{Beta}\left(c_{j}(y), d_{j}(y)\right)
\end{aligned}
$$

The modified parameters are calculated by setting equal $(\doteq)$ the first and second moments of $\tilde{p}\left(\vartheta_{i} \mid Y_{i j}=y\right), \tilde{p}\left(\varrho_{j} \mid Y_{i j}=y\right)$ to the real marginal distributions $p\left(\vartheta_{i} \mid Y_{i j}=y\right), p\left(\varrho_{j} \mid Y_{i j}=y\right)$, respectively.

We list the results in [12] as follows.

$$
\begin{aligned}
\mathbb{E}_{1}\left(\vartheta_{i}\right) & =\frac{a_{i}\left(\left(c_{j}\left(a_{i}+1\right)+b_{i} d_{j}\right)\right)}{\left(a_{i}+b_{i}+1\right)\left(a_{i} c_{j}+b_{i} d_{j}\right)} \\
\mathbb{E}_{0}\left(\vartheta_{i}\right) & =\frac{a_{i}\left(\left(d_{j}\left(a_{i}+1\right)+b_{i} c_{j}\right)\right)}{\left(a_{i}+b_{i}+1\right)\left(b_{i} c_{j}+a_{i} d_{j}\right)} \\
\mathbb{E}_{1}\left(\vartheta_{i}^{2}\right) & =\frac{a_{i}\left(a_{i}+1\right)\left(\left(a_{i}+2\right) c_{j}+b_{i} d_{j}\right)}{\left(a_{i}+b_{i}+1\right)\left(a_{i}+b_{i}+2\right)\left(a_{i} c_{j}+b_{i} d_{j}\right)} \\
\mathbb{E}_{0}\left(\vartheta_{i}^{2}\right) & =\frac{a_{i}\left(a_{i}+1\right)\left(\left(a_{i}+2\right) d_{j}+b_{i} c_{j}\right)}{\left(a_{i}+b_{i}+1\right)\left(a_{i}+b_{i}+2\right)\left(b_{i} c_{j}+a_{i} d_{j}\right)}
\end{aligned}
$$

Similarly, we can calculate $c_{j}(y), d_{j}(y)$ the same way. 